3. Панченко В.А., Дубровин В.С.. Механика. Теоретическая механика, техническая механика. Учебное пособие, Москва: Российский университет транспорта (МИИТ), 2018. ISBN 978-5-7473-0875-6, 191 с.

4. Панченко В.А., Синицын С.А., Дубровин В.С.. Выполнение сборочных моделей и рабочей конструкторской документации средствами САПР КОМПАС-3D. Учебное пособие, Москва: Российский университет транспорта (МИИТ), 2018. ISBN 978-5-7473-0918-0, 164 с.

5. Гусарова О.Ф., Панченко В.А., Синицын С.А. Инженерная компьютерная гафика. РРУТ, Москва, 2020, 149c.

6. Погорелов И.А., Гурский Ф.А., Панченко В.А. Разработка трекера для солнечных модулей. Инновации в сельском хозяйстве. 2018, №2(27), с.226-231.

7. Панченко В.А. Моделирование солнечных теплофотоэлектрических модулей // Электротехнологии и электрооборудование в АПК, 2019, 2 (35), с. $71-77$.

\title{
Синицын С.A. \\ Применение информационных оценок для построения адекватных моделей проектирования в итерационных системах
}

Российский университет транспорта (РУТ(МИИТ))

(Россия, Москва)

doi: 10.18411/trnio-11-2021-52

\section{Аннотация}

В основе метода информационного проектирования заложена диапазонная схема параметризации. Здесь каждый проектный параметр определен величиной доверительного диапазона и законом распределения вероятностей своей реализации внутри этого диапазона. В классическом методе проектирования на каждом этапе вычисляется некоторая центральная оценка параметра, которая принимается за единственно верную в дальнейшем проектировании. В диапазонной схеме все параметры внутри доверительного диапазона принимаются в качестве достоверных для дальнейшего решения с некоторой вероятностью, соответствующей закону распределения. Поскольку доверительный диапазон определяется величиной и законом распределения вероятностей, то его совокупной оценкой может быть аддитивная характеристика - энтропия, которая лишена размерности в отличии от проектных параметров. Свойство аддитивности безразмерной энтропии позволяет выполнять простое арифметическое суммирование параметров модели с целью получения ее интегральной характеристики, которая показывает степень адекватности модели процессу проектирования. Тогда эффективность процесса проектирования на этапе может быть оценена приростом количества информации по уменьшению энтропии.

Ключевые слова: итерационное проектирование, проектные параметры, доверительные диапазоны, распределения вероятностей, энтропия параметра, адекватность модели, погрешности проектирования.

\section{Abstract}

The information design method is based on a range parameterization scheme. Here, each design parameter is determined by the value of the confidence range and the law of the probability distribution of its implementation within this range. In the classical design method, at each stage, a certain central estimate of the parameter is calculated, which is taken as the only correct one in further design. In the range scheme, all parameters within the confidence range are taken as reliable for further decisions with a certain probability corresponding to the distribution law. Since the confidence range is determined by the magnitude and the law of probability distribution, its cumulative estimate can be an additive characteristic - entropy, which is dimensionless in contrast to the design parameters. The additivity property of dimensionless entropy makes it possible to perform a simple arithmetic summation of the model parameters in order to obtain its integral characteristics, which shows the degree of model adequacy to the design process. Then the efficiency of the design process at the stage can be estimated by the increase in the amount of information on the reduction of entropy.

Keywords: iterative design, design parameters, confidence ranges, probability distributions, parameter entropy, model adequacy, design errors. 
Наиболее сложным и важным вопросом математического моделирования в САПР является оценка степени адекватности модели объекту проектирования. Адекватность отражает полноту соответствия формализованного математического описания реальному объекту или процессу. На практике понятие адекватности, как правило, заменяется термином точности модели в широком смысле. Определение степени адекватности модели достаточно сложная задача, которая может быть решена достаточно точно лишь при наличии реальных физических объектов, например, на заключительных стадиях проектирования. Соответственно на ранних стадиях, таких как этапы технических предложений, проектных решений, объектов сравнения нет [1, с.53].

Теоретически каждому проектному решению можно было бы поставить в соответствие физическую модель, но такой подход сделал бы проект баснословно дорогим. Тем более, что вариантов проектных решений может быть два и более.

Математические модели систем проектирования создаются по блочному принципу. Минимальной единицей модели может быть блок или модуль из разряда типовых, входящих в состав банка данных для моделирования. Потребность создания моделей заданной адекватности продиктовала необходимость систематизировать модели одной функциональной группы по показателям точности. В дальнейшем эту характеристику будем идентифицировать с более широким понятием качества модуля или самой расчетной модели в целом.

Таким образом, задача оценки степени адекватности расчетной модели этапа проектирования сводится к оценкам точности отдельных функциональных модулей по выбранному единому признаку [2, с.75]. Правильное решение этой задачи определяет область достоверных решений на каждом этапе проектирования.

Задача оценки достоверности проектных параметров традиционно решается на основе средних оценок, например математического ожидания без учета параметров доверительного диапазона возможных решений. Нетрудно предположить, что значительное количество вполне рациональных решений выбрасывается из дальнейшего алгоритма проектирования. Такая оценка решений, как правило, приводит к ложным выводам при принятии решений, а сама модель считается не пригодной по точности расчетов.

Предложенный нами метод информационного проектирования позволяет оценивать проектные решения на всем диапазоне возможных решений с учетом распределения вероятностей по всему диапазону. Такая характеристика называется энтропией Шеннона и является некоторой суммарной весовой функцией всех точек кривой закона распределения. В этом случае эффективная погрешность будет определена энтропией, а степень адекватности модели процессу проектирования - разностью энтропий, то есть приростом количества информации.

Применение энтропийной погрешности позволяет учитывать некоторое множество величин проектных параметров из доверительного диапазона достоверности расчетной модели с учетом величины и закона распределения параметров на самом диапазоне [3,c.122]. Только тогда, с заданной вероятностью, ни одно из возможных правильных решений не будет потеряно.

Известные обзоры и систематизация многообразия методов оценки точности преобразователей информации, к которым мы можем отнести и математические модели нашего класса, дают рекомендации о практических методах оценки достоверности результатов преобразований.

Как правило, выделяются две компоненты неточности: неопределенность и погрешность. Погрешность, как систематическая составляющая неточности, складывается из трех составляющих: функциональной погрешности; метрической, связанной с выбором единицы измерения; погрешности аппроксимации в широком понимании, в том числе технических компромиссов.

Более правильно характеристику неточности определять двумя универсальными составляющими - случайной и систематической. Систематическая составляющая может 
быть постоянной или меняться по некоторому известному закону. Минимальная систематическая ошибка всегда определяет максимальную точность расчетной модели, то есть ее адекватность процессу проектирования. К сожалению случайные ошибки, в некоторых случаях, исключают действительные результаты, поэтому их нужно исключать или хотя бы минимизировать.

С другой стороны, все погрешности моделирования можно разделить на две основные группы: принципиально-теоретические и инструментальные. К первой группе относятся ошибки от использования приближенных математических зависимостей, на основе которых выполняется большинство проектных расчетов. Эти ошибки известны заранее и могут быть учтены с помощью поправочных таблиц и графиков [4,c.118]. Инструментальные погрешности включают также случайные ошибки, которых, как уже говорилось, следует избегать.

Наиболее правильное представление о природе формирования погрешностей в моделях САПР можно получить, если представить некоторый преобразователь в виде черного ящика, на вход которого воздействует сигнал $\mu$, а на выходе принимается сигнал $\mu^{\prime}$, который принимает значения в некотором диапазоне $\Delta \mu^{\prime}$. При этом значение $\mu^{\prime}$ является одним из вероятных в заданном диапазоне.

Подавляющее наличие систематических погрешностей моделей проектирования заставляет еще раз обратить внимание на энтропийную погрешность. Которая не имеет физической размерности, обладает свойствами аддитивности и, как уже говорилось выше, характеризует доверительный диапазон вместе с законом распределения вероятностей реализаций проектных параметров [5,с.229].

Энтропийная погрешность позволяет произвести точную количественную оценку самых разных моделей проектирования с целью их сравнения на этапе формирования из отдельных модулей с единой информационной точки зрения. Очевидно, что для определения степени развития той или иной системы проектирования невозможно пользоваться ни пространственным, ни массово-энергетическим критерием. Так, например, нельзя утверждать, что тот функциональный блок математической модели проектирования дает меньшие информационные потери, который «больше размером» или дольше рассчитывается с помощью компьютера. Объективный информационный критерий способен системно оценивать степень развития проекта при переходе от этапа к этапу в безразмерном пространстве проектных параметров. Возможность сравнения различных групп проектных параметров по содержанию информации столь же естественна, как сравнение однородных тел по объему [6, с.74].

Анализ возможностей информационного подхода для формирования процесса проектирования позволяет сделать вывод об эффективности метода не только при изучении характеристик и свойств блочных моделей проектирования, но и для широкого класса задач многопараметрического моделирования в САПР.

Помимо задач проектирования, управляемое решение многомерного моделирования позволяет регулировать точность математических моделей, применяемых в различных технологических процессах и прочих системах оптимального управления на основе математического моделирования.

$$
* * *
$$

1. Гусарова О.Ф., Панченко В.А., Синицын С.А. Инженерная компьютерная гафика. РРУТ, Москва, 2020, 149с.

2. Панченко В.А. Моделирование солнечных теплофотоэлектрических модулей // Электротехнологии и электрооборудование в АПК, 2019, 2 (35), с. $71-77$.

3. Панченко В.А., Синицын С.А., Дубровин В.С. Выполнение сборочных моделей и рабочей конструкторской документации средствами САПР Компас-3D. РУТ, Москва, 2018, 164c.

4. Левчук Т.В., Дубровин В.С., Панченко В.А. Механика, теоретическая механика и пррикладная механика. РУТ, Москва, 2021, 190с.

5. Погорелов И.А., Гурский Ф.А., Панченко В.А. Разработка трекера для солнечных модулей. Инновации в сельском хозяйстве. 2018, №2(27), с.226-231.

6. Панченко В.А. Моделирование солнечных теплофотоэлектрических модулей // Электротехнологии и электрооборудование в АПК, 2019, 2 (35), с. $71-77$. 